
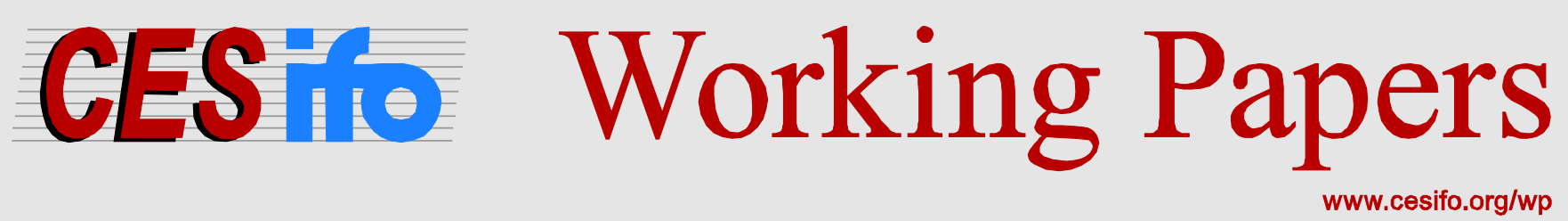

\title{
Optimal Favoritism in All-Pay Auctions and Lottery Contests
}

\author{
Jörg Franke \\ Wolfgang Leininger \\ Cédric Wasser
}

CESIFO WORKING PAPER NO. 6274

CATEGORY 11: INDUSTRIAL ORGANISATION

DECEMBER 2016
An electronic version of the paper may be downloaded
- from the SSRN website: Www.SSRN.com
- from the RePEc website: Www.RePEc.org
- from the CESifo website: www.CESifo-group.org/wp




\title{
Optimal Favoritism in All-Pay Auctions and Lottery Contests
}

\begin{abstract}
We analyze the revenue-enhancing potential of favoring specific contestants in complete information all-pay auctions and lottery contests with several heterogeneous contestants. Two instruments of favoritism are considered: Head starts that are added to the bids of specific contestants and multiplicative biases that give idiosyncratic weights to the bids. In the all-pay auction, head starts are more effective than biases while optimally combining both instruments even yields first-best revenue. In the lottery contest, head starts are less effective than biases and combining both instruments cannot further increase revenue. As all-pay auctions revenuedominate lottery contests under optimal biases, we thus obtain an unambiguous revenue-ranking of all six combinations of contest formats and instruments.
\end{abstract}

JEL-Codes: C720, D720.

Keywords: all-pay auction, lottery contest, favoritism, head start, revenue dominance.

\author{
Jörg Franke \\ University of Dortmund (TU) \\ Department of Economics \\ Vogelpothsweg 87 \\ Germany - 44227 Dortmund \\ joerg.franke@tu-dortmund.de
}

\author{
Wolfgang Leininger \\ University of Dortmund (TU) \\ Department of Economics \\ Vogelpothsweg 87 \\ Germany-44227 Dortmund \\ wolfgang.leininger@tu-dortmund.de
}

\author{
Cédric Wasser \\ University of Bonn \\ Department of Economics \\ Lennéstr. 37 \\ Germany - 53113 Bonn \\ cedric.wasser@uni-bonn.de
}

December, 2016

This paper supersedes Ruhr Economic Paper No. 524: "Revenue Maximizing Head Starts in Contests”. The authors would like to thank audiences at various seminars, workshops and conferences for helpful comments. Jörg Franke is grateful to Dan Kovenock for helpful discussions and his hospitality during a research visit at the Economic Science Institute at Chapman University. 


\section{Introduction}

Contests are frequently and increasingly used to allocate scarce resources among competing agents when other allocation mechanism like markets, matching, or bargaining protocols are not feasible, impractical, or not desired. A characteristic feature of contests is that participating agents exert effort or pay non-refundable bids to win an indivisible prize such that all agents incur their respective costs of effort exertion irrespectively of winning the prize or not. Examples range from promotion tournaments within firms to lobbying, from public procurement to rent-seeking, from high school admission to crowdsourcing, and from the allocation of research grants to innovation contests; see Konrad (2009),[26], and Vojnović (2016), [39], for excellent textbooks on contest theory and their applications, as well as Frank and Cook (2010), [15], and English (2005), [10], for popular approaches regarding the related phenomenon of winner-take-all-markets and the ubiquity of contests in arts and culture.

As the organizer of a contest typically has substantial discretionary power in designing the contest rules, there is a tendency for explicit or implicit favoritism with respect to specific agents. Consider, for instance, the preferential treatment of internal or external candidates in hiring decisions or of domestic or small business firms in public procurement, handicap systems in sports, affirmative action in high school admission, or simply discrimination in the sense that the contest organizer favors specific contestants which is manifested by tailoring the conditions of the contest to the advantage of the preferred contestants. In all these cases agents are treated asymmetrically, which might have profound implications for the underlying incentive structure of the contest.

Often, these forms of asymmetric treatment can be interpreted as either granting head starts or as biasing the contest rule in favor of specific agents. Agents that enjoy head starts benefit from their advantageous position in the sense that their rivals must first pass the head start to be able to compete on equal footing. Agents that are favored by a biased contest rule enjoy a higher weight on their effort in the process of determining the winner of the prize. ${ }^{1}$ Naturally, the extent of head starts and bias has strategic implications for favored agents, their respective rivals, and therefore also on the revenue that is generated in the contest. A contest organizer who has the option to fit bias and/or head starts to the underlying heterogeneity of the contestants can

\footnotetext{
${ }^{1}$ The University of Michigan, for example, added a head start of 20 (out of 150) points to the score of minority applicants for their undergraduate program, while small business firms are granted a 5 percent bid discount in California highway procurement auctions, see Krasnokutskaya and Seim (2011), [27]. There are also instances where both instruments are applied simultaneously: Kirkegaard (2012), [23], reports on the Canadian research promotion program, where researchers with excellent past performance receive a head start while the research proposals of junior scientists get a higher weight in the evaluation process.
} 
therefore influence the generated contest revenue to some degree.

In this paper we focus on these two instruments of favoritism (bias and head starts) and analyze their potential to generate additional revenue in contest games with several heterogeneous players. We concentrate on two frameworks that are predominantly used in the contest literature; that is, all-pay auctions with complete information, see Hillman and Riley (1989), [20], for an early analysis, and lottery contests introduced by Tullock (1980), [38]. Both models are sufficiently tractable and have been extensively applied in various contexts, see the two mentioned text books and Corchón (2007), [6] for a survey. However, our model deviates from the standard setup by allowing the contest organizer to specify idiosyncratic head starts and/or biases; that is, granting additive lump-sum boni (head starts) or weighting the respective bids or effort levels with different factors (bias). Hence, applying both instruments simultaneously amounts to an affine transformation of the bid or effort. From the perspective of a contest organizer who is interested in maximizing aggregated equilibrium effort or bids (the expected revenue of the contest), specifying the framework-specifc optimal bias and/or head start becomes then the crucial instrument to increase contest revenue. ${ }^{2}$

Our analysis proceeds by characterizing the equilibrium for a given bias-head-start combination for each of the two frameworks. For the lottery contest we modify the share-function approach by Wasser (2013), [40], to obtain an indirect characterization of equilibrium revenue. We then consider an unbiased lottery framework with head starts to show that head starts in general are not very effective in inducing additional revenue in a lottery contest: Basically, favored players use their head start to substitute for own effort while other players are not affected, which tends to decrease equilibrium revenue. Moreover, for each feasible head start vector there exists an appropriate bias that induces higher revenue. Hence, in the lottery contest framework the optimal bias revenue-dominates the optimal head start and the optimal combination of bias and head starts involves zero head starts.

For the all-pay auction framework with head starts and heterogeneous players there does not exist a complete characterization of the equilibrium set. Hence, we take a more indirect approach by identifying lower bounds on revenue, based on a restricted class of all-pay auctions where head starts are specified such that only two players are active in equilibrium. Based on this class we characterize optimal head starts, the revenue-maximizing set of active players, and the respectively induced expected revenue in equilibrium. This value constitutes our lower bound on

\footnotetext{
${ }^{2}$ Arguably, in some of the applications mentioned above, asymmetric treatment of agents is not (at least officially) implemented to increase contest revenue but rather for some normative reasons. However, even if normatively derived deviations from symmetric treatment are applied, the forgone revenue should be an important evaluation criterion for these policies. Our paper provides a benchmark of comparison by deriving the maximal revenue that can be obtained through optimally designing asymmetric treatment of agents.
} 
revenue for the unrestricted case where more than two players might be active. We also show that in general this lower bound is not tight as in some settings there exist alternative head starts with three active players that lead to even higher revenue. If bias and head starts are applied simultaneously, then the optimal bias-head-start combination mimics a take-it-or-leave-it offer to the player with the highest valuation in the sense that this player faces a rival with a head start equal to her valuation. Using an appropriate tie-breaking rule and bias weights, the strongest player is forced to bid exactly her valuation to overcome the high head start which implies that the entire surplus is extracted from the players and transformed one-to-one into additional revenue. Thus, in the all-pay auction framework head starts are highly effective instruments for revenue extraction and their efficiency is even stronger if combined with appropriately designed biases.

Finally, we compare the generated contest revenue within and between the two frameworks under the optimally designed instruments. We obtain a complete and unconditional revenueranking among all frameworks and instrument combinations, which holds for any degree of heterogeneity among the players. The resulting ranking implies, for instance, that revenue in the all-pay auction under optimal head starts is higher than under the optimal bias. Hence, head starts are specifically effective to induce additional revenue if the contest is highly competitive as in the all-pay auction, where strong players have to surpass the head start of their rivals to maintain their chance of winning the prize. Multiplicative biases, by contrast, are more effective in the lottery contest because they can be designed to induce additional entry by weak players which tends to increases revenue, comp. Franke et al. (2013), [17]. In the lottery contest this mechanism does not work with additive head starts because favored players use head starts as a substitute for own effort exertion. Nevertheless, the all-pay auction revenue-dominates the lottery contest independently of the fact whether the respective optimal head start, or the optimal bias, or both are used in the two frameworks, generalizing the revenue dominance result of Franke et al. (2014), [18], to any affine bid transformation. ${ }^{3}$ Hence, if revenue maximization is the objective of the contest organizer, then she should resort to the all-pay auction framework with optimal bias-head-start combination.

Our study contributes to the literature on revenue maximization with biased contest success functions and heterogeneous players, comp. Nti (2004), [33], Fu (2006), [19], Franke (2012), [16], and Epstein et al. (2011 and 2013), [11] and [12]; see also Mealem and Nitzan (forthcoming), [31], for a recent survey on discrimination in contest games. ${ }^{4}$ Moreover, our study

\footnotetext{
${ }^{3}$ In the discussion section we show that the ranking is also robust with respect to concave bid transformation functions.

${ }^{4}$ Similar issues have also been analyzed experimentally, comp. Balafoutas and Sutter (2012), [2], Niederle et al. (2013), [32], and Calsamiglia et al. (2013), [5].
} 
contributes to the recent interest in the analysis of head starts in different competitive situations, comp. Kirkegaard (2012), [23], Seel and Wasser (2014), [35], Li and Yu (2012), [28], Segev and Sela (2014), [36], Konrad (2002), [25], as well as Imhof and Kräkel (2016), [21]. However, all of the mentioned studies (as most of the literature) are either restricted to the two-player case or focus exclusively on only one of the two instruments. In this sense our characterization of the optimal head start in the multi-player all-pay auction framework constitutes, for instance, a direct extension of $\mathrm{Li}$ and $\mathrm{Yu}$ (2012), [28], to the multi-player case, while a similar relation holds for the lottery contest analyzed in Nti (2004), [33]. To our knowledge there only exist three studies that involve head starts in multi-player all-pay auction or lottery contest frameworks with heterogeneous players. ${ }^{5}$ While Siegel (2014), [37], considers head starts in multi-prize all-pay auctions, Wasser (2013), [40], incorporates homogeneous head starts in a lottery contest framework. However, both studies are rather interested in equilibrium characterization and not in the optimal head start design. The only paper that explicitly addresses the design of optimal head starts and biases in a multi-player lottery contest framework is Dasgupta and Nti (1998), [8]. However, they assume that players and head starts are homogeneous and find that in their specific case no head start at all is optimal. Our study extends this framework by considering heterogeneous contestants with idiosyncratic head starts and biases and shows under which conditions these previously established results on optimal favoritism still hold under heterogeneity.

The paper is organized as follows. In Section 2 we introduce the model setup and the two contest frameworks. In Section 3 we characterize the equilibrium in the lottery contest framework with bias and head starts. Based on this characterization we derive the optimal head starts, summarize the results on the optimal bias and analyze the simultaneous application of bias and head starts. We collect our result for the lottery contest and provide a complete revenue ranking of all instrument combinations in this framework. In Section 4 we analyze the all-pay auction framework by deriving a lower bound on equilibrium revenue based on all-pay auctions with optimal head starts where only two player are active. We then demonstrate that this lower bound is higher than expected revenue under the optimally biased all-pay auction. Finally, we identify the optimal combination of bias and head starts and show that it is equivalent to a take-it-or-leave-it offer to the strongest player which therefore yields revenue at the upper bound. In Section 5 we collect our results to compare equilibrium revenue between the two frameworks and provide a complete revenue ranking among all instrument combinations and both frameworks. In Section

\footnotetext{
${ }^{5}$ There is also a recent literature on the optimality of favoritism among ex-ante symmetric players when the organizer cannot observe each player's strength, see Drugov and Ryvkin (forthcoming), [9], Pérez-Castrillo and Wettstein (2016), [34], Matros and Possajennikov (2016), [30], and Kawamura and Moreno de Barreda (2014), [22].
} 
6 we discuss the robustness of our results with respect to alternative instruments of favoritism and more general bid transformations. Section 7 concludes.

\section{The Model}

There are $n \geq 2$ players of set $\mathcal{N}=\{1, \ldots, n\}$ that compete for an indivisible prize. Players are heterogeneous with respect to their valuations of the prize and can be ordered decreasingly with respect to their valuations: $v_{1} \geq v_{2} \geq \ldots \geq v_{n}>0$. The probability $\operatorname{Pr}_{i}\left(x_{i}, x_{-i}\right)$ of player $i \in \mathcal{N}$ to win the prize depends positively on her bid $x_{i} \in[0, \infty)$ and negatively on the bids $x_{-i}=\left(x_{1}, \ldots, x_{i-1}, x_{i+1}, \ldots, x_{n}\right) \in[0, \infty)^{n-1}$ of her rivals, where all bids are non-refundable. The expected payoff for player $i \in \mathcal{N}$ is

$$
\pi_{i}\left(x_{i}, x_{-i}\right):=\operatorname{Pr}_{i}\left(x_{i}, x_{-i}\right) v_{i}-x_{i}
$$

The probability function $\operatorname{Pr}_{i}\left(x_{i}, x_{-i}\right)$ depends on the specific design of the contest framework. We focus in our analysis on the two most frequently used contest frameworks; that is, a deterministic all-pay auction and a probabilistic lottery contest. More precisely, we consider asymmetric versions of those two contest frameworks where the bid $x_{i}$ of each player $i \in \mathcal{N}$ is converted by an idiosyncratic affine transformation consisting of a multiplicative bias parameter $\alpha_{i} \in[0, \infty)$ and a non-negative head start $\delta_{i} \in[0, \infty)$ that is added to player $i$ 's bid without any costs. Once, the decision on the framework is made and the respective instrument combination of bias $\alpha=\left(\alpha_{1}, \ldots, \alpha_{n}\right)$ and head starts $\delta=\left(\delta_{1}, \ldots, \delta_{n}\right)$ is designed (given the specific framework), the probability for player $i \in \mathcal{N}$ to win the prize can be expressed as follows:

1. For the all-pay auction with bias and head starts (framework BHA),

$$
\operatorname{Pr}_{i}^{B H A}\left(x_{i}, x_{-i}\right):= \begin{cases}1 & \text { if } \alpha_{i} x_{i}+\delta_{i}>\alpha_{j} x_{j}+\delta_{j} \text { for all } j \neq i, \\ \tau_{i}(\mathcal{M}) & \text { if } i \in \mathcal{M}=\left\{m \in \mathcal{N}: \alpha_{m} x_{m}+\delta_{m} \geq \alpha_{j} x_{j}+\delta_{j} \forall j \in \mathcal{N}\right\} \\ & \text { and }|\mathcal{M}| \geq 2, \\ 0 & \text { if } \alpha_{i} x_{i}+\delta_{i}<\alpha_{j} x_{j}+\delta_{j} \text { for some } j \neq i,\end{cases}
$$

where the tie-breaking rule $\tau$ satisfies $\tau_{i}(\mathcal{K}) \in[0,1]$ and $\sum_{i \in \mathcal{K}} \tau_{i}(\mathcal{K})=1$ for all $i \in \mathcal{K}$ and $\mathcal{K} \subseteq \mathcal{N}$. If not stated otherwise, our results below hold for all tie-breaking rules $\tau$. The fair tie-breaking rule $\tau^{f}$, which is prevalent in the literature, is defined as $\tau_{i}^{f}(\mathcal{K}):=\frac{1}{|\mathcal{K}|}$ for all $i \in \mathcal{K}$ and $\mathcal{K} \subseteq \mathcal{N}$. 
2. For the lottery contest with bias and head starts (framework BHL),

$$
\operatorname{Pr}_{i}^{B H L}\left(x_{i}, x_{-i}\right):= \begin{cases}\frac{\alpha_{i} x_{i}+\delta_{i}}{\sum_{j=1}^{n}\left(\alpha_{j} x_{j}+\delta_{j}\right)} & \text { if } \sum_{j=1}^{n}\left(\alpha_{j} x_{j}+\delta_{j}\right) \neq 0 \\ 0 & \text { if } \sum_{j=1}^{n}\left(\alpha_{j} x_{j}+\delta_{j}\right)=0\end{cases}
$$

These probability functions are also called contest success functions (CSFs). It should be noted, that they include two special cases that are of interest in their own right for our analysis: Applying a symmetric bias to all players, e.g., $\alpha=(1, \ldots, 1)$, leads to an unbiased all-pay auction/lottery contest with head starts (framework HA/HL); applying zero head starts to all players, $\delta=(0, \ldots, 0)$, leads to a biased all-pay auction/lottery contest without head starts (framework $\mathrm{BA} / \mathrm{BL})$.

We evaluate the revenue potential of the two instruments by comparing the maximal contest revenue (the sum of expected equilibrium bids by all players) that can be induced in each framework by specifying optimal head starts and/or biases. We denote this maximal revenue by $X_{F}^{*}:=\sum_{i \in \mathcal{N}} E\left[x_{F, i}^{*}\right]$, where $E\left[x_{F, i}^{*}\right]$ denotes the expected equilibrium bid of player $i \in \mathcal{N}$ under an optimal bias and/or head start in framework $F \in\{B H A, B H L, H A, H L, B A, B L\}$. Analyzing all $2 \times 3$ frameworks separately allows us to isolate the revenue-enhancing effects of the two instruments in separation and to compare them with the optimal affine transformation where both instruments are used simultaneously.

As bias and head starts are framework-specific, our analysis proceeds by firstly characterizing optimal head starts, optimal biases, and the optimal affine transformation for each framework. These instruments are then compared with respect to the induced revenue within each framework and, in a last step, between the two frameworks. ${ }^{6}$

\section{The Lottery Contest}

Our analysis of the lottery contest framework starts with an explicit characterization of the equilibrium for a given head-start-bias combination $(\delta, \alpha)$. We then determine optimal head starts without bias (framework HL) and summarize the existing results concerning optimal biases without head starts (framework BL). Finally, we show that the optimal combination of head starts and bias (framework BHL) coincides with the optimal biases with zero head starts. Hence, using head starts in addition to bias is not conducive to generate additional revenue in a lottery contest. In

\footnotetext{
${ }^{6}$ Our analysis can be alternatively framed as the design problem of a contest organizer, whose objective function depends positively on contest revenue, and whose strategy space consists of the type of contest framework with type-dependent instrument (i.e., bias and/or head starts).
} 
other words, a revenue-maximizing contest organizer would choose to specify optimal biases and refrain from using head starts at all.

\subsection{Equilibrium}

Consider a lottery contest with head starts $\delta \in[0, \infty)^{n}$ and bias $\alpha \in[0, \infty)^{n}$, i.e., the most general framework BHL. Note that for players $j$ with $\alpha_{j}=0$ investing positive effort is strictly dominated by zero effort. Hence, define $\tilde{\mathcal{N}}:=\left\{i \in \mathcal{N}: \alpha_{i}>0\right\}$ to be the set of potentially active players.

Let $y_{i}:=\alpha_{i} x_{i}+\delta_{i}$ denote the score of player $i \in \mathcal{N}$ and $Y:=\sum_{i \in \mathcal{N}} y_{i}$ the aggregate score. We will consider the equivalent game where each player $i \in \tilde{\mathcal{N}}$ chooses his score $y_{i} \in\left[\delta_{i}, \infty\right)$ rather than his effort $x_{i}$ and obtains payoff

$$
\tilde{\pi}_{i}\left(y_{1}, \ldots, y_{n}\right)=\frac{y_{i}}{Y} v_{i}-\frac{y_{i}-\delta_{i}}{\alpha_{i}} \quad(\text { if } Y>0) \text {. }
$$

As $\tilde{\pi}_{i}$ is strictly concave in $y_{i}$, player $i$ 's best response is characterized by the first-order condition

$$
\frac{Y-y_{i}}{Y^{2}} v_{i}-\frac{1}{\alpha_{i}} \leq 0, \quad \text { with equality if } y_{i}>\delta_{i}
$$

An effort profile $x_{1}, \ldots, x_{n}$ in the original contest is a pure-strategy Nash equilibrium if and only if the corresponding score profile $y_{1}, \ldots, y_{n}$ satisfies (1) for all $i \in \tilde{\mathcal{N}}$ and $x_{j}=0$ for all $j \notin \tilde{\mathcal{N}}$.

In the following, we generalize the equilibrium characterization of Wasser (2013), [40], who considered lottery contests with symmetric head starts and without bias and made use of the share function approach by Cornes and Hartley (2005), [7].

For all $\mathcal{K} \subseteq \tilde{\mathcal{N}}$, define the function

$$
Y(\mathcal{K}):= \begin{cases}\frac{|\mathcal{K}|-1+\sqrt{(|\mathcal{K}|-1)^{2}+4\left(\sum_{i \in \mathcal{K}} \frac{1}{\alpha_{i} v_{i}}\right)\left(\sum_{j \notin \mathcal{K}} \delta_{j}\right)}}{2 \sum_{i \in \mathcal{K}} \frac{1}{\alpha_{i} \nu_{i}}} & \text { if } \mathcal{K} \neq \varnothing, \\ \sum_{i \in \mathcal{N}} \delta_{i} & \text { if } \mathcal{K}=\varnothing .\end{cases}
$$

This function from the power set of $\tilde{\mathcal{N}}$ to $\mathbb{R}_{+}$is central to our equilibrium characterization: As shown in the following proposition, maximizing $Y(\mathcal{K})$ with respect to $\mathcal{K}$ yields the equilibrium aggregate score, with the maximizer being the set of active players that submit non-zero effort in equilibrium. 
Proposition 3.1 There is a unique pure-strategy Nash equilibrium in the BHL framework. Let

$$
\mathcal{K}^{*}=\underset{\mathcal{K} \subseteq \tilde{\mathcal{N}}}{\arg \max } Y(\mathcal{K})
$$

In equilibrium, the aggregate score is $Y\left(\mathcal{K}^{*}\right)$, each player $i \in \mathcal{K}^{*}$ exerts effort

$$
x_{i}=\frac{1}{\alpha_{i}}\left(Y\left(\mathcal{K}^{*}\right)-\frac{Y\left(\mathcal{K}^{*}\right)^{2}}{\alpha_{i} v_{i}}-\delta_{i}\right)>0
$$

and each player $j \in \mathcal{N} \backslash \mathcal{K}^{*}$ exerts zero effort.

Proof. Note that condition (1) implies $y_{i}=\max \left\{Y-\frac{Y^{2}}{\alpha_{i} v_{i}}, \delta_{i}\right\}$. Following Cornes and Hartley (2005), [7], we define $i$ 's share function as

$$
\phi_{i}(Y):=\frac{y_{i}}{Y}= \begin{cases}\max \left\{f_{i}(Y), g_{i}(Y)\right\} & \text { if } i \in \tilde{\mathcal{N}} \\ g_{i}(Y) & \text { if } i \notin \tilde{\mathcal{N}}\end{cases}
$$

where $f_{i}(Y):=1-\frac{Y}{\alpha_{i} v_{i}}$ and $g_{i}(Y):=\frac{\delta_{i}}{Y}$. Let $\Phi(Y):=\sum_{i=1}^{n} \phi_{i}(Y)$ be the aggregate share function.

A score profile $y_{1}, \ldots, y_{n}$ corresponds to a pure-strategy Nash equilibrium if and only if there is an aggregate score $Y^{*}$ such that $y_{i}=\phi_{i}\left(Y^{*}\right) Y^{*}$ for each $i$ and $\Phi\left(Y^{*}\right)=1$. Note that for players $i$ who are active in equilibrium $\left(x_{i}>0\right.$ and $\left.y_{i}>\delta_{i}\right)$ we have $\phi_{i}\left(Y^{*}\right)=f_{i}\left(Y^{*}\right)$, whereas for inactive players $\left(x_{i}=0\right.$ and $\left.y_{i}=\delta_{i}\right)$ we have $\phi_{i}\left(Y^{*}\right)=g_{i}\left(Y^{*}\right)$.

$\Phi(Y)$ is continuous and strictly decreasing. Moreover, $\Phi\left(\sum_{j} \delta_{j}\right) \geq \sum_{i} g_{i}\left(\sum_{j} \delta_{j}\right)=1$ and $\Phi(Y)<1$ for $Y$ large enough. Hence, there is a unique $Y^{*}$ that solves $\Phi\left(Y^{*}\right)=1$, implying existence of a unique pure-strategy Nash equilibrium.

Now, consider an equilibrium where the players in $\mathcal{K}^{*} \subseteq \tilde{\mathcal{N}}$ are active, whereas the remaining players are inactive. Hence, $\Phi\left(Y^{*}\right)=1$ is equivalent to

$$
\Phi\left(Y^{*}\right)=\sum_{i \in \mathcal{K}^{*}} f_{i}\left(Y^{*}\right)+\sum_{j \notin \mathcal{K}^{*}} g_{j}\left(Y^{*}\right)=\left|\mathcal{K}^{*}\right|-Y \sum_{i \in \mathcal{K}^{*}} \frac{1}{\alpha_{i} v_{i}}+\frac{1}{Y^{*}} \sum_{j \notin \mathcal{K}^{*}} \delta_{j}=1 .
$$

Solving this equation for $Y^{*}$ we obtain $Y^{*}=Y\left(\mathcal{K}^{*}\right)$, where $Y(\cdot)$ was defined in (2).

We will now show that the function $Y(\mathcal{K})$ is maximized at the set of players $\mathcal{K}^{*}$ that are active in equilibrium. Consider a set of players $\mathcal{M} \subseteq \tilde{\mathcal{N}}$ such that $\mathcal{M} \neq \mathcal{K}^{*}$. By the definition of $Y(\cdot)$, we have

$$
\sum_{i \in \mathcal{M}} f_{i}(Y(\mathcal{M}))+\sum_{j \notin \mathcal{M}} g_{j}(Y(\mathcal{M}))=1
$$


However, since $\mathcal{M}$ does not correspond to the set of players that are active in equilibrium, we must have at least one $i \in \mathcal{M}$ where $f_{i}(Y(\mathcal{M}))<g_{i}(Y(\mathcal{M}))$ or one $j \notin \mathcal{M}$ where $g_{j}(Y(\mathcal{M}))<$ $f_{j}(Y(\mathcal{M}))$. Hence, $\Phi(Y(\mathcal{M}))>1$. Since $\Phi$ is strictly decreasing, we must have $Y(\mathcal{M})<Y\left(\mathcal{K}^{*}\right)$, because $\mathcal{K}^{*}$ is the unique subset of players that satisfies $\Phi\left(Y\left(\mathcal{K}^{*}\right)\right)=1$.

Finally, the equilibrium efforts of active players $i \in \mathcal{K}^{*}$ given in (3) are obtained from the equilibrium scores $y_{i}=\phi_{i}\left(Y\left(\mathcal{K}^{*}\right)\right) Y\left(\mathcal{K}^{*}\right)=Y\left(\mathcal{K}^{*}\right)-\frac{Y\left(\mathcal{K}^{*}\right)^{2}}{\alpha_{i} v_{i}}$.

Proposition 3.1 establishes existence and uniqueness of a pure-strategy Nash equilibrium for any combination of head starts and bias and provides a characterization of equilibrium efforts based on the aggregate score. This characterization will prove useful for studying the optimal use of head starts and bias by the contest organizer. Before determining the revenue-maximizing head-start-bias combination, we will proceed by first considering each of the two instruments separately.

\subsection{Optimal head starts}

We will determine optimal head starts within framework HL by setting $\alpha=(1, \ldots, 1)$ and taking advantage of the equilibrium characterization provided by Proposition 3.1. To clarify the dependence on head starts $\delta$, we will slightly change the notation from the preceding subsection: We will write $Y(\delta, \mathcal{K})$ for the function defined in $(2), \mathcal{K}^{*}(\delta)$ for the set of players that are active in equilibrium, and $x_{i}(\delta)$ for the equilibrium effort of player $i$.

The revenue induced under head starts $\delta$ without bias simplifies to the equilibrium aggregate score minus the sum of the head starts. Making use of Proposition 3.1, we have

$$
X_{H L}(\delta):=\sum_{i \in \mathcal{N}} x_{i}(\delta)=Y\left(\delta, \mathcal{K}^{*}(\delta)\right)-\sum_{i \in \mathcal{N}} \delta_{i}
$$

An important benchmark is the revenue in the standard lottery contests without head starts. We will represent this case by the zero head start vector $\delta_{0}:=(0, \ldots, 0)$. The induced revenue simplifies to

$$
X_{H L}\left(\delta_{0}\right)=Y\left(\delta_{0}, \mathcal{K}^{*}\left(\delta_{0}\right)\right)=\max _{\mathcal{K} \subseteq \mathcal{N}} Y\left(\delta_{0}, \mathcal{K}\right)=\max _{k>1} \frac{k-1}{\sum_{i=1}^{k} \frac{1}{v_{i}}} .
$$

As a first step towards finding optimal head starts, the following lemma provides an upper bound on revenue $X_{H L}(\delta)$, which depends on the number of players that are active in equilibrium.

Lemma 3.2 Let $\delta \neq \delta_{0}$. If $\left|\mathcal{K}^{*}(\delta)\right|=1$, then $X_{H L}(\delta) \leq \frac{1}{4} v_{1}$. If $\left|\mathcal{K}^{*}(\delta)\right| \geq 2$, then $X_{H L}(\delta)<X_{H L}\left(\delta_{0}\right)$. 
Proof. First, suppose $\delta$ is such that only one player is active, i.e., $\mathcal{K}^{*}(\delta)=\{i\}$ for some $i$. Then

$$
X_{H L}(\delta)=Y(\delta,\{i\})-\sum_{j \in \mathcal{N}} \delta_{j}=\sqrt{v_{i} \sum_{j \neq i} \delta_{j}}-\sum_{j \neq i} \delta_{j}-\delta_{i} \leq \sqrt{\sum_{j \neq i} \delta_{j}}\left(\sqrt{v_{i}}-\sqrt{\sum_{j \neq i} \delta_{j}}\right) \leq \frac{v_{i}}{4} \leq \frac{v_{1}}{4} .
$$

The first inequality is implied by $\delta_{i} \geq 0$, the second inequality follows from the expression on the LHS being maximized at $\sqrt{\sum_{j \neq i} \delta_{j}}=\frac{1}{2} \sqrt{v_{i}}$, and the third inequality uses $v_{1} \geq v_{i}$ for all $i$.

Now, suppose $\delta$ induces $\left|\mathcal{K}^{*}(\delta)\right| \geq 2$. Observe that for any $\mathcal{K} \subseteq \mathcal{N}$ with $|\mathcal{K}| \geq 2$, we have

$$
\frac{\partial Y(\delta, \mathcal{K})}{\partial \delta_{i}}= \begin{cases}0 & \text { if } i \in \mathcal{K} \\ \frac{1}{\sqrt{(|\mathcal{K}|-1)^{2}+4\left(\sum_{l \in \mathcal{K}} \frac{1}{v_{l}}\right)\left(\sum_{j \notin \mathcal{K}} \delta_{j}\right)}} \leq \frac{1}{|\mathcal{K}|-1} \leq 1 & \text { if } i \notin \mathcal{K}\end{cases}
$$

where $\frac{\partial Y(\delta, \mathcal{K})}{\partial \delta_{i}}<1$ for $i \notin \mathcal{K}$ with $\delta_{i}>0$. Hence, $\frac{\partial}{\partial \delta_{j}}\left(Y(\delta, \mathcal{K})-\sum_{i \in \mathcal{N}} \delta_{i}\right) \leq 0$ for all $j \in \mathcal{N}$, with strict inequality for at least one $j$. This leads to the following chain of (in-)equalities, which proves the second result:

$$
X_{H L}(\delta)=Y\left(\delta, \mathcal{K}^{*}(\delta)\right)-\sum_{i \in \mathcal{N}} \delta_{i}<Y\left(\delta_{0}, \mathcal{K}^{*}(\delta)\right) \leq Y\left(\delta_{0}, \mathcal{K}^{*}\left(\delta_{0}\right)\right)=X_{H L}\left(\delta_{0}\right)
$$

Lemma 3.2 differentiates between two cases. If at least two players are active under head starts $\delta$, then revenue can be increased by removing any individual head start $\delta_{i}$ irrespective of whether player $i$ is active or inactive. For active players, head starts are perfect substitutes for own effort because (1) implies that the best-response score of an active player will not be affected by a reduction of his head start. For inactive players, the proof of Lemma 3.2 reveals that reducing their head starts is also revenue-enhancing. Hence, zero head starts $\delta_{0}$ induce higher revenue than any other vector of head starts that result in at least two active players.

If, however, exactly one player is active under head starts $\delta$, then setting positive head starts for inactive players can be beneficial. Intuitively, increasing the head starts of inactive players may lead the active player to best-respond by increasing his effort. Lemma 3.2 shows that revenue in this case is bounded above by $\frac{1}{4} v_{1}$.

The existence of this upper bound has an immediate implication. If revenue without head starts is higher than $\frac{1}{4} v_{1}$, then Lemma 3.2 implies that optimal head starts are zero. In the opposite case, the following result shows that there exist head starts under which revenue meets the upper bound, i.e., player 1 submits effort $\frac{1}{4} v_{1}$ and all other players remain inactive. 
Proposition 3.3 If $\frac{1}{4} v_{1} \geq X_{H L}\left(\delta_{0}\right)$, then all head starts $\delta^{*}$ where $\delta_{1}^{*}=0$ and $\sum_{j=2}^{n} \delta_{j}^{*}=\frac{1}{4} v_{1}$ are optimal and induce revenue $X_{H L}\left(\delta^{*}\right)=x_{1}\left(\delta^{*}\right)=\frac{1}{4} v_{1}$. Otherwise, zero head starts $\delta_{0}$ are the unique optimal head starts.

Proof. Suppose $\frac{1}{4} v_{1} \geq X_{H L}\left(\delta_{0}\right)$. We will show that any head starts $\delta^{*}$ as defined in the proposition result in equilibrium efforts $x_{1}\left(\delta^{*}\right)=\frac{1}{4} v_{1}$ and $x_{j}\left(\delta^{*}\right)=0$ for all $j>1$. The aggregate score in such an equilibrium is $Y=x_{1}\left(\delta^{*}\right)+\sum_{j=2}^{n} \delta_{j}^{*}=\frac{1}{2} v_{1}$. Recall that player $i$ 's best response in terms of individual score is given by (1), which implies

$$
y_{i}=\max \left\{Y\left(1-\frac{Y}{v_{i}}\right), \delta_{i}^{*}\right\}=\max \left\{\frac{1}{2} v_{1}\left(1-\frac{v_{1}}{2 v_{i}}\right), \delta_{i}^{*}\right\} .
$$

Hence, the best-response effort of player 1 is indeed $x_{1}=y_{1}=\frac{1}{4} v_{1}$. Moreover, recall (4) and note that $\frac{1}{4} v_{1} \geq X_{H L}\left(\delta_{0}\right)=\max _{k>1} \frac{k-1}{\sum_{i=1}^{k} \frac{1}{v_{i}}} \geq \frac{1}{\frac{1}{v_{1}}+\frac{1}{v_{2}}}$ implies $3 v_{2} \leq v_{1}$. Therefore, $\frac{v_{1}}{2 v_{j}} \geq \frac{3}{2}$ for all $j>1$, implying $y_{j}=\delta_{j}^{*}$. Consequently, the best-response effort of each player $j>1$ is indeed $x_{j}=0$.

Now, suppose $\frac{1}{4} v_{1}<X_{H L}\left(\delta_{0}\right)$. In this case, Lemma 3.2 implies that $X_{H L}\left(\delta_{0}\right)>X_{H L}(\delta)$ for all $\delta \neq \delta_{0}$, rendering $\delta_{0}$ uniquely optimal.

Proposition 3.3 implies that non-zero head starts are optimal if player 1's valuation is sufficiently higher than the other players' valuations. A simple sufficient condition for $\frac{1}{4} v_{1} \geq X_{H L}\left(\delta_{0}\right)$ is $v_{1} \geq 4 v_{2}$ (whereas $v_{1} \geq 3 v_{2}$ is necessary, as shown in the proof of Proposition 3.3). If non-zero head starts are optimal, then they are set such that the strongest contestant, player 1, competes against the sum of the head starts of all the other players, which are inactive. Hence, from the perspective of player 1 it is as if he were facing just one equally strong opponent. If, however, the difference in valuations between player 1 and the other players is less pronounced, then head starts are not a suitable instrument for increasing revenue.

Using (4), Proposition 3.3 implies the following for revenue under optimal head starts.

Corollary 3.4 Optimal head starts in the HL framework yield revenue

$$
X_{H L}^{*}=\max \left\{\frac{v_{1}}{4}, \max _{k>1} \frac{k-1}{\sum_{i=1}^{k} \frac{1}{v_{i}}}\right\} .
$$

\subsection{Optimal biases}

We now turn to framework BL, where the organizer can only set a bias $\alpha$ but no head starts, (i.e., the zero head start vector $\delta=\delta_{0}=(0, \ldots, 0)$ is pre-specified). The revenue-maximizing bias for 
this case has been determined in Franke et al. (2013), [17], which is summarized in the following proposition.

Proposition 3.5 (Franke et al. (2013), [17]) An optimal bias in the BL framework yields revenue

$$
X_{B L}^{*}=\frac{1}{4}\left(\sum_{i=1}^{k^{*}} v_{i}-\frac{\left(k^{*}-2\right)^{2}}{\sum_{i=1}^{k^{*}} \frac{1}{v_{i}}}\right), \quad \text { where } \quad k^{*}=\max \left\{k \in \mathcal{N} \mid \frac{k-2}{v_{k}}<\sum_{i=1}^{k} \frac{1}{v_{i}}\right\}
$$

The set of optimal biases consists of all $\alpha^{*}$ where, for some $c>0$,

$$
\alpha_{i}^{*}=2 c\left(v_{i}+\frac{k^{*}-2}{\sum_{j=1}^{k^{*}} \frac{1}{v_{j}}}\right)^{-1} \text { for } i \leq k^{*} \quad \text { and } \quad \alpha_{i}^{*}<\frac{c}{v_{i}} \quad \text { for } i>k^{*}
$$

Under an optimal bias, each player $i \leq k^{*}$ is active and each player $i>k^{*}$ is inactive.

In an optimally biased lottery contest, the $k^{*}$ contestants with the highest valuations are active in equilibrium. Compared with the unbiased lottery contest, an optimal bias $\alpha^{*}$ typically encourages additional entry $\left(k^{*}\right.$ is greater or equal to the number of active players in an unbiased lottery contest). For example, provided that $n \geq 3$, at least three players will always be active in the optimally biased lottery contest while this lower bound is two in the unbiased contest. In this respect, the way in which optimal biases work is in stark contrast to our characterization of optimal head starts from the preceding subsection: If non-zero head starts are optimal, then they are designed to discourage entry, reducing the number of active players to one.

\subsection{Optimal combinations of head starts and bias}

We now consider lottery contests where both instruments, head starts and bias, can be used simultaneously. Our main result for this case is that an organizer will never find it profitable to implement non-zero head starts in addition to an optimally chosen bias. In other words, any contest with head starts and bias can be replaced by a contest without head starts and adjusted bias that yields strictly higher revenue.

Consider a contest with strictly positive head starts for some players and suppose we remove them. Each player $i$ that is active under a positive head start will increase his effort due to the fact that head starts are perfect substitutes for own effort exertion. In addition, we prove that by manipulating $\alpha_{j}$, each inactive player $j$ can be induced to actively invest effort that makes up for the removed head start. 
Proposition 3.6 For any combination of head starts and bias $(\delta, \alpha)$ where $\delta \neq \delta_{0}$, there exists a bias $\hat{\alpha}$ such that the contest with $\left(\delta_{0}, \hat{\alpha}\right)$ yields strictly higher revenue than the contest with $(\delta, \alpha)$.

Proof. Let $y_{1}, \ldots, y_{n}$ denote the equilibrium scores induced under $(\delta, \alpha)$ where $\delta \neq \delta_{0}$ and recall $Y=\sum_{i \in \mathcal{N}} y_{i}$. Hence, $y_{1}, \ldots, y_{n}$ are the unique scores that satisfy (1) for each $i \in \tilde{\mathcal{N}}$. Moreover, let $\mathcal{K}^{*}(\delta, \alpha)$ denote the set of players that are active in this equilibrium (i.e., all $i$ where $y_{i}>\delta_{i}$ ). Define a new bias $\hat{\alpha}$ such that $\hat{\alpha}_{i}=\alpha_{i}$ for all $i \in \mathcal{K}^{*}(\delta, \alpha)$ and $\hat{\alpha}_{j}=\frac{Y^{2}}{\left(Y-\delta_{j}\right) v_{j}}>0$ for all $j \notin \mathcal{K}^{*}(\delta, \alpha)$.

We will now show that each individual equilibrium score $\hat{y}_{i}$ under $\left(\delta_{0}, \hat{\alpha}\right)$ is identical to the equilibrium score $y_{i}$ under $(\delta, \alpha)$. Adapting $(1), \hat{y}_{1}, \ldots, \hat{y}_{n}$ are the equilibrium scores under $\left(\delta_{0}, \hat{\alpha}\right)$ if they satisfy, for each $i \in \mathcal{N}$,

$$
\frac{\hat{Y}-\hat{y}_{i}}{\hat{Y}^{2}} v_{i}-\frac{1}{\hat{\alpha}_{i}} \leq 0, \quad \text { with equality if } \hat{y}_{i}>0 .
$$

Hence, we have to show that $\hat{y}_{i}=y_{i}$ for each $i$ (and thus $\hat{Y}=Y$ ) solves (5).

For the originally active players $i \in \mathcal{K}^{*}(\delta, \alpha)$, we have $\hat{\alpha}=\alpha$ and hence the fact that $y_{1}, \ldots, y_{n}$ solve (1) for $i$ immediately implies that the same scores also solve (5) for $i$. For the originally inactive players $j \notin \mathcal{K}^{*}(\delta, \alpha)$, condition (5) becomes

$$
\frac{\hat{Y}-\hat{y}_{j}}{\hat{Y}^{2}} v_{j}-\frac{Y-\delta_{j}}{Y^{2}} v_{j} \leq 0, \quad \text { with equality if } \hat{y}_{j}>0 .
$$

Noting that $y_{j}=\delta_{j}$, the above is indeed satisfied for $\hat{Y}=Y$ and $\hat{y}_{j}=y_{j}$.

Let $x_{i}$ and $\hat{x}_{i}$ denote $i$ 's equilibrium effort under $(\delta, \alpha)$ and $\left(\delta_{0}, \hat{\alpha}\right)$, respectively. Since $y_{i}=\hat{y}_{i}$, $\hat{x}_{i}=x_{i}+\frac{\delta_{i}}{\hat{\alpha}_{i}}$ for all $i \in \mathcal{N}$. Consequently, $\sum_{i \in \mathcal{N}} \hat{x}_{i}>\sum_{i \in \mathcal{N}} x_{i}$ because $\delta_{i}>0$ for at least one $i$.

The crucial implication of Proposition 3.6 is, that an optimal bias $\alpha^{*}$ as provided by Proposition 3.5 combined with zero head starts is also optimal in framework BHL, resulting in maximal revenue $X_{B H L}^{*}=X_{B L}^{*}$. Summarizing our results, we obtain the following revenue ranking for the different instruments in the lottery contest framework.

Proposition 3.7 There is an unambiguous revenue-ranking of lottery contests with optimal head starts and/or biases: If $v_{1}=v_{2}$, then

$$
X_{B H L}^{*}=X_{B L}^{*} \geq X_{H L}^{*}
$$

If $v_{1}>v_{2}$, then

$$
X_{B H L}^{*}=X_{B L}^{*}>X_{H L}^{*}
$$


Proof. The equalities and the weak inequality are immediate from Proposition 3.6. It remains to prove the strict inequality $X_{B L}^{*}>X_{H L}^{*}=\max \left\{\frac{1}{4} v_{1}, X_{H L}\left(\delta_{0}\right)\right\}$ if $v_{1}>v_{2}$. If $X_{H L}^{*}=\frac{1}{4} v_{1}$, then according to Proposition 3.3 at least one head start is strictly positive and the strict inequality follows from Proposition 3.6. If $X_{H L}^{*}=X_{H L}\left(\delta_{0}\right)$, then the strict inequality follows from the fact that $X_{B L}^{*}$ is strictly higher than the revenue in absence of any bias if $v_{1}>v_{2}$. To see this, note that if $v_{1}>v_{2}$, Proposition 3.5 implies $\alpha_{1}^{*}<\alpha_{2}^{*}$ for each optimal bias $\alpha^{*}$. Applying no bias $(\alpha=(1, \ldots, 1))$ is hence not optimal and yields strictly lower revenue.

From the perspective of a revenue-maximizing contest organizer biasing the contest rule is the preferred instrument of favoritism. Under the optimal bias more contestants are induced to participate and the playing field is more balanced. Both effects imply higher effort exertion and therefore higher revenue. By contrast, head starts are less suitable to induce additional revenue because contestants who are favored by positive head starts use them to reduce their effort level while maintaining their score. In equilibrium the score of other contestants is therefore not affected and revenue is generically lower under positive head starts.

Moreover, for any head start there exists a corresponding bias that yields even higher revenue by inducing non-active contestants to participate. As this substitution works for any degree of heterogeneity in valuations, the respective revenue ranking is unambiguous and confirms the dominance of optimal biases over head starts in the lottery contest framework.

\section{The All-Pay Auction}

Turning to the all-pay auction, we proceed as follows. After stating some preliminary results, we determine and discuss bounds on the revenue generated by optimal head starts in framework HA. Then, we summarize the existing results on optimal biases in framework BA and identify optimal head-start-bias combinations in framework BHA. Our results allow us to compare all instruments with respect to induced revenue which leads to an unambiguous ranking of the three instrument combinations with respect to their potential for revenue extraction in the all-pay auction. In isolation, head starts turn out to be more conducive to generating revenue than biases while combining the two instruments even allows for extracting first-best revenue at the upper bound. 


\subsection{Preliminaries}

We start with two preliminary observations. First, we show that the highest valuation $v_{1}$ represents a general upper bound on the revenue that can be obtained in an all-pay auction with head starts and bias. Second, we easily obtain from the analysis of Baye et al. (1993), [3], that this upper bound is reached in a standard all-pay auction if $v_{1}=v_{2}$, implying that using no head starts and no bias maximizes revenue in this case.

Lemma 4.1 For all head starts $\delta$ and biases $\alpha$, the induced revenue is at most $v_{1}$.

$$
\text { If } v_{1}=v_{2} \text {, then } X_{B H A}^{*}=X_{H A}^{*}=X_{B A}^{*}=v_{1} \text {. }
$$

Proof. Note that by investing zero effort, each player can always ensure himself a payoff of at least zero. Hence, in every Nash equilibrium a player's expected effort cannot exceed his valuation times the probability that he wins. The sum of players' valuations weighted with their winning probabilities therefore is an upper bound on revenue, which is less than or equal to $v_{1}$.

Now, suppose $v_{1}=v_{2}$ and consider the standard all-pay auction without head starts and bias, i.e., $\alpha=(1, \ldots, 1)$ and $\delta=(0, \ldots, 0)$. Then Theorem 1 in Baye et al. (1993), [3], implies that every Nash equilibrium yields revenue $v_{1}$. Consequently, the first-best revenue can be obtained in all three frameworks by applying no head starts and no bias.

Having established that in all three frameworks the optimal use of the instruments yields first-best revenue if $v_{1}=v_{2}$, we will focus on $v_{1}>v_{2}$ in the following.

\subsection{Optimal head starts}

Let us turn to studying optimal head starts in framework HA where any multiplicative bias is absent such that we can set $\alpha=(1, \ldots, 1)$, for example. For the case of two players, revenuemaximizing head starts in the all-pay auction have been determined by $\mathrm{Li}$ and $\mathrm{Yu}$ (2012), [28]. They show that it is optimal to assign to the weaker player 2 a head start of $v_{1}-v_{2}$ over the stronger player 1 . In equilibrium, player 1 then randomizes his effort uniformly on $\left[v_{1}-v_{2}, v_{1}\right]$ and player 2 chooses zero effort with probability $\frac{v_{1}-v_{2}}{v_{1}}$ while randomizing uniformly on $\left[0, v_{2}\right]$ otherwise, resulting in an expected payoff of zero for both players. We record their results in the following lemma.

Lemma 4.2 (Li and Yu (2012), [28]) If $n=2$, then head starts $\delta_{1}^{*}=0$ and $\delta_{2}^{*}=v_{1}-v_{2}$ are 
optimal in framework HA and induce revenue

$$
X_{H A}^{*}=v_{1}-\frac{v_{2}}{2}+\frac{v_{2}^{2}}{2 v_{1}} .
$$

Compared with the standard all-pay auction without head starts, revenue under optimal head starts for $n=2$ is higher because the support of player 1's equilibrium effort distribution is shifted upwards by $\delta_{2}^{*}=v_{1}-v_{2}$ while player 2's equilibrium strategy remains unchanged. Player 1's increase in effort thus exactly offsets player 2's head start, implying that the same total surplus is realized as in the standard all-pay auction. Under optimal head starts, however, this total surplus is entirely collected by the contest organizer. Put differently, the optimal head starts transform player 1's entire payoff of $v_{1}-v_{2}$ from the standard all-pay auction into additional revenue.

Now, suppose $n>2$. Note that in the all-pay auction, the organizer can use head starts to exclude any number of players from the contest: Whenever $\delta_{j}-\delta_{i}>v_{i}$ for some $j$, player $i$ will stay inactive in any equilibrium. One possibility is hence to exclude all but two players from the contest and to set the relative head starts of those two players as in Lemma 4.2. If the identity of those two players is also chosen optimally, then this results in the optimal head starts within the class of head starts with exactly two players being active in equilibrium. The induced revenue represents a lower bound for the revenue under optimal head starts in framework HA. We state this lower bound in the following proposition, where we also show that there exist no head starts that induce first-best revenue $v_{1}$ if $v_{1}>v_{2}$.

Proposition 4.3 Revenue under optimal head starts in framework HA satisfies

$$
X_{H A}^{*} \geq v_{1}-\frac{\min \left\{v_{2}\left(v_{1}-v_{2}\right), v_{n}\left(v_{1}-v_{n}\right)\right\}}{2 v_{1}}= \begin{cases}v_{1}-\frac{v_{2}}{2}+\frac{v_{2}^{2}}{2 v_{1}} & \text { if } v_{1}<v_{2}+v_{n}, \\ v_{1}-\frac{v_{n}}{2}+\frac{v_{n}^{2}}{2 v_{1}} & \text { if } v_{1}>v_{2}+v_{n} .\end{cases}
$$

Moreover, $X_{H A}^{*}<v_{1}$ if $v_{1}>v_{2}$.

Proof. Consider two players $\{i, j\} \subseteq \mathcal{N}$ such that $i<j$ and let the head starts be $\delta_{i}=e$, $\delta_{j}=v_{i}-v_{j}+e$, and $\delta_{k}=0$ for all $k \notin\{i, j\}$, where $e>v_{1}$. Under these head starts, all players $k \notin\{i, j\}$ remain inactive whereas players $i$ and $j$ compete like in a two-player contest with optimal head starts $\left(\delta_{j}-\delta_{i}=v_{i}-v_{j}\right.$ as in Lemma 4.2), resulting in revenue $X(i, j):=v_{i}-\frac{v_{j}}{2}+\frac{v_{j}^{2}}{2 v_{i}}$.

The lower bound stated in the proposition corresponds to $\max _{\{i, j\}} X(i, j)$. To see this, note that $\frac{\partial X(i, j)}{\partial v_{i}}=1-\frac{1}{2}\left(\frac{v_{j}}{v_{i}}\right)^{2}>0$, which implies that it is optimal to set $i=1$. Moreover, as $\frac{\partial X(1, j)}{\partial v_{j}}=\frac{v_{j}}{v_{1}}-\frac{1}{2}$, revenue $X(1, j)$ is decreasing in $v_{j}$ for low values of $v_{j}$ and increasing for high values. Hence, 
either $j=2$ or $j=n$ is optimal, i.e.,

$$
\max _{\{i, j\}} X(i, j)=\max \{X(1,2), X(1, n)\}=v_{1}-\frac{\min \left\{v_{2}\left(v_{1}-v_{2}\right), v_{n}\left(v_{1}-v_{n}\right)\right\}}{2 v_{1}} .
$$

The statement in the proposition holds because $v_{2}\left(v_{1}-v_{2}\right)<v_{n}\left(v_{1}-v_{n}\right)$ if and only if $v_{1}<v_{2}+v_{n}$.

It remains to prove that there are no head starts that induce first-best revenue $v_{1}$ if $v_{1}>v_{2}$. First note that if $v_{1}>v_{2}$, revenue $v_{1}$ can only be obtained in an equilibrium where player 1 invests effort $v_{1}$ and wins with probability one. Such an equilibrium exists only if each player $j>1$ has no incentive to outbid player 1, i.e., only if $v_{1}+\delta_{1} \geq v_{j}+\delta_{j}$. A necessary condition is hence that head starts satisfy $v_{1}-v_{n} \geq \delta_{j}-\delta_{1}$ for all $j>1$. But then player 1 would deviate from bidding $v_{1}$ because any bid $x_{1} \in\left(v_{1}-v_{n}, v_{1}\right)$ would also result in winning with probability one.

The optimal head starts within the class of $\delta$ with exactly two active players used for the lower bound in Proposition 4.3 let the strongest player compete against either the second strongest or the weakest player, depending on whether $v_{1}<v_{2}+v_{n}$ or $v_{1}>v_{2}+v_{n}$. Let $j$ denote the player who competes against player 1. Under the optimal head starts of Lemma 4.2, player 1's expected effort $v_{1}-\frac{v_{j}}{2}$ is decreasing in the weaker player's valuation $v_{j}$ because a lower $v_{j}$ allows for a higher head start $\delta_{j}-\delta_{1}=v_{1}-v_{j}$ that encourages player 1 to bid more aggressively. By contrast, player $j$ 's expected effort $\frac{v_{j}^{2}}{2 v_{1}}$ is increasing in $v_{j}$. If $v_{1}<v_{2}+v_{n}$, i.e., if player 1 's valuation is relatively low, then the second effect dominates the first. Hence, it is optimal to make $v_{j}$ as large as possible by selecting the second strongest player as player 1's active opponent. If player 1's valuation is relatively high, however, the first effect outweighs the second such that it is optimal to let him compete against the weakest player.

The lower bound on revenue in Proposition 4.3 will prove sufficient for obtaining a general revenue ranking of the different instrument combinations. However, one may wonder whether this lower bound is tight or whether an organizer could do strictly better by implementing head starts under which three or more players are active in equilibrium. The following result shows that the latter is indeed possible.

Proposition 4.4 Suppose $n=3, v_{1}>v_{2}>v_{3}$, and $v_{1}<v_{2}+v_{3}$. Then, for all $\gamma \in\left(0, \frac{v_{2}\left(v_{1}-v_{2}\right)\left(v_{2}-v_{3}\right)}{v_{1} v_{3}}\right]$, the all-pay auction with head starts $\delta_{1}=0, \delta_{2}=v_{1}-v_{2}, \delta_{3}=\delta_{2}+\gamma$ has an equilibrium with three active players that induces revenue

$$
X_{H A}>v_{1}-\frac{v_{2}}{2}+\frac{v_{2}^{2}}{2 v_{1}} .
$$


Proof. See the Appendix.

Proposition 4.4 implies that whenever $n \geq 3$ and $v_{1}<v_{2}+v_{n}$, there are head starts that render three players active and generate strictly more revenue than the optimal head starts within the class of $\delta$ with exactly two active players. Hence, not even in a generic sense - as one might have expected - is it true that two active players in equilibrium can yield the optimal revenue. The fact that maximizing revenue within framework HA may require more than two players actively competing is in stark contrast to optimal biases within framework BA (discussed below) or optimal exclusion in the standard all-pay auction (Baye et al., 1993, [3]), where two active players are always sufficient.

Note that a complete characterization of the set of equilibria of all-pay auctions with head starts is still an open question. With Proposition 4.4 suggesting the need for considering equilibria with three or more active players, a general characterization of optimal head starts is hence challenging and beyond the scope of this paper. $^{7}$

\subsection{Optimal bias}

The biased all-pay auction without head starts (framework BA) has been extensively analyzed in Franke et al. (2014), [18], where the optimal bias and the corresponding maximal revenue have been identified following a similar approach like in the previous subsection; that is, deriving a lower bound on equilibrium revenue by using the restricted class with two active players where a unique equilibrium in closed form exists. As in the previous subsection the optimal bias in framework BA neutralizes the difference in valuations among the two active agents. However, in contrast to framework HA the two strongest players will always be active under the optimal bias. Moreover, the lower bound thereby obtained is also an upper bound, implying the following result for maximal revenue in framework BA.

Proposition 4.5 (Franke et al. (2014), [18]) An optimal bias in the BA framework yields equilibrium revenue $X_{B A}^{*}=\frac{v_{1}+v_{2}}{2}$.

Using the results from Proposition 4.3 and 4.5 we are now in a position to evaluate the revenue extraction potential of the two instruments by comparing the respectively induced revenue. The

\footnotetext{
${ }^{7}$ While Siegel (2014), [37], provides important results on all-pay auctions with head starts, they cannot be applied here as the head starts in both Lemma 4.2 and Proposition 4.4 violate his genericity assumption. Moreover, in our single-prize framework the algorithm of Siegel (2014), [37], is restricted to situations where exactly two players are active in equilibrium.
} 
following proposition implies that head starts are more effective than biases in an all-pay auction framework, which can be attributed to the cut-throat competition in the all-pay auction: If head starts are present in the all-pay auction then every active player must bid higher than the maximal head start among all players because all other bids are strictly dominated. Hence, head starts are powerful instruments for revenue extraction in an all-pay auction context.

Proposition 4.6 If $v_{1}>v_{2}$, optimal head starts in framework HA strictly revenue dominate optimal biases in framework $B A: X_{H A}^{*}>X_{B A}^{*}$.

Proof. From Proposition 4.3 we know that $X_{H A}^{*} \geq \frac{v_{2}^{2}}{2 v_{1}}+v_{1}-\frac{v_{2}}{2}$. Hence it is sufficient to show that $\frac{v_{2}^{2}}{2 v_{1}}+v_{1}-\frac{v_{2}}{2}>\frac{v_{1}+v_{2}}{2}=X_{B A}^{*}$. This inequality can be reduced to $\left(v_{1}-v_{2}\right)^{2}>0$, which always holds.

\subsection{Optimal combinations of head starts and bias}

We now consider revenue-maximizing affine transformations of bids, where non-negative head starts are combined with multiplicative biases (framework BHA). While we showed in the previous subsection that head starts are more effective than biases when used in isolation, the question remains whether combining the two instruments allows for even higher revenue extraction. In contrast to the lottery contest the answer is affirmative in the all-pay auction: Optimal affine transformations of bids yield first-best revenue $v_{1}$.

Proposition 4.7 With an appropriately chosen tie-breaking rule $\tau$, optimal combinations of head starts and bias in the BHA framework yield revenue $X_{B H A}^{*}=v_{1}$.

Proof. Suppose the tie-breaking rule is such that $\tau_{1}(\mathcal{M})=1$ whenever $1 \in \mathcal{M}$. Moreover, let $\alpha_{1}=1, \delta_{1}=0, \alpha_{2}=0, \delta_{2}=v_{1}$, and $\alpha_{j}=\delta_{j}=0$ for $j>2$. In the unique Nash equilibrium, player 1 invests effort $v_{1}$ and wins with certainty while all other players invest zero effort.

By combining head starts and bias with an appropriate tie-breaking rule, the contest organizer can extract first-best revenue $v_{1}$ by mimicking an optimal take-it-or-leave-it offer to the strongest player: The organizer approaches the strongest player and offers her the prize if she matches a rival's head start equal to her valuation, while keeping all other players inactive by setting their bias parameters equal to zero.

Note that Proposition 4.7 requires that the organizer is able to manipulate the tie-breaking rule in favor of player 1 . However, if the organizer cannot design $\tau$ and is restricted to use the 
fair tie-breaking rule $\tau^{f}$ or any other specific tie-breaking rule, he can still implement the firstbest revenue in the limit. As the following result shows, revenue $v_{1}$ can be approached arbitrarily closely with head starts and biases that induce player 1 to choose effort randomly but close to $v_{1}$ and a second player to be active with a small probability, resulting in ties happening with probability zero.

Proposition 4.8 Suppose $v_{1}>v_{2}$. For any tie-breaking rule $\tau$ and any $\varepsilon \in\left(0, \frac{v_{1}-v_{2}}{2}\right)$, there is a combination of head starts and bias that yields revenue $v_{1}-\varepsilon$.

Proof. For all tie-breaking rules $\tau$, revenue $v_{1}-\varepsilon$ can be implemented as follows. Set $\delta_{1}=0$, $\alpha_{1}=1, \delta_{2}=v_{1}\left(1-\frac{2 \varepsilon}{v_{1}-v_{2}}\right)$, and $\alpha_{2}=\frac{2 \varepsilon v_{1}}{\left(v_{1}-v_{2}\right) v_{2}}$, and $\alpha_{j}=\delta_{j}=0$ for $j>2$. In the unique Nash equilibrium, player 1 randomizes uniformly on $\left[\delta_{2}, v_{1}\right]$ and player 2 bids zero with probability $\frac{\delta_{2}}{v_{1}}$ and with the remaining probability randomizes uniformly on $\left[0, v_{2}\right]$. All other player remain inactive. This yields revenue $\frac{\delta_{2}+v_{1}}{2}+\left(1-\frac{\delta_{2}}{v_{1}}\right) \frac{v_{2}}{2}=v_{1}-\varepsilon$.

In contrast to the lottery contest, optimal bias-head-start combinations in the all-pay auction are not used to increase competitive pressure by encouraging additional entry of players. Instead, they allow the contest organizer to manipulate the CSF of the all-pay auction in such a way that competition is basically reduced to the strongest player who has to compete against an opponent backed by a head start which is equal (or arbitrarily close) to her valuation. As a result, the payoff of the strongest player is zero and revenue equals (or approaches arbitrarily closely) the first best.

Finally, combining Proposition 4.7 with Propositions 4.6 and 4.3 as well as Lemma 4.1 yields a complete revenue ranking of all combinations of instruments in the all-pay auction framework.

Corollary 4.9 There is an unambiguous revenue-ranking of all-pay auctions with optimal head starts and/or biases: If $v_{1}=v_{2}$, then

$$
X_{B H A}^{*}=X_{H A}^{*}=X_{B A}^{*} .
$$

If $v_{1}>v_{2}$, then

$$
X_{B H A}^{*}>X_{H A}^{*}>X_{B A}^{*}
$$

Proof. The equalities in (6) are from Lemma 4.1. The first inequality in (7) follows from Proposition 4.7 and the last statement in Proposition 4.3. The second inequality in (7) is stated in Proposition 4.6. 


\section{Revenue Ranking}

The separate analysis of the all-pay auction and the lottery contest framework facilitated a withinframework comparison of the different instruments with respect to induced revenue in Proposition 3.7 and Corollary 4.9. It remains to complete the revenue comparison between the two frameworks under the respective instruments. The following result shows that the all-pay auction with optimal bias and/or head start revenue-dominates any lottery contest irrespectively of the used instruments. More importantly, we thus obtain an unambiguous revenue-ranking of all six combinations of instruments and frameworks, which holds for any degree of heterogeneity among contestants.

Proposition 5.1 There is an unambiguous revenue-ranking of all-pay auctions and lottery contests with optimal head starts and/or biases: If $v_{1}=v_{2}$, then

$$
X_{B H A}^{*}=X_{H A}^{*}=X_{B A}^{*}>X_{B L}^{*}=X_{B H L}^{*} \geq X_{H L}^{*}
$$

If $v_{1}>v_{2}$, then

$$
X_{B H A}^{*}>X_{H A}^{*}>X_{B A}^{*}>X_{B L}^{*}=X_{B H L}^{*}>X_{H L}^{*}
$$

Proof. The first two and the last two relations in each line are implied by Proposition 3.7 and Corollary 4.9. The remaining inequality $X_{B A}^{*}>X_{B L}^{*}$ is implied by the revenue-dominance theorem in Franke et al. (2014), [18].

Proposition 5.1 has two important implications: Firstly, it shows that the all-pay auction with optimal bias-head-start combination revenue-dominates any symmetric or asymmetrically biased all-pay auction or lottery contest and any all-pay auction or lottery contest with or without head starts. Secondly, the revenue-dominance of the (optimally biased) all-pay auction over the lottery contest, established in Franke et al. (2014), [18], also holds more generally for alternative instruments like optimal head starts or optimal affine bid transformations. ${ }^{8}$ Hence, a contest organizer interested in maximizing revenue should always resort to the all-pay auction framework as long as at least one of the mentioned instruments can be applied.

\footnotetext{
${ }^{8}$ The fact that these revenue rankings do not depend on the heterogeneity of the contestants is in contrast to the unbiased frameworks without head starts where no such unambiguous ranking exists, comp. Fang (2002), [14].
} 


\section{Discussion}

Our analysis is focused on additive head starts and multiplicative biases as instruments for favoritism in contest design. In this section we discuss the robustness of our results by considering alternative instruments and more general bid transformations. Entry fees, minimum bids, or handicaps (i.e., negative head starts) are pertinent examples for alternative instruments of contest design which allow the contest organizer to extract additional revenue from the contestants in a rather direct way. In a complete information setting like ours, ${ }^{9}$ they can be designed in an obvious way to extract revenue at the upper bound in both frameworks: If entry fees, minimum bids, or handicaps are set arbitrarily close to the valuations of the strongest player, then all but the strongest player drop out of the contest, while the strongest player is forced to bid her valuation. Hence, under optimal use these alternative instruments yield first-best revenue both in the all-pay auction and in the lottery contest framework.

A more interesting extension is to allow the contest organizer to design positive and increasing score functions that need not be affine. As the all-pay auction with optimal affine score functions already induces equilibrium revenue at the upper bound, non-affine score functions cannot improve upon the optimal bias-head-start combination in the all-pay auction framework. However, in the lottery contest framework the question remains whether non-affine score functions could induce higher revenue than the optimal affine ones.

Without any further restrictions on score functions this is indeed the case. Using a family of convex score functions, the organizer can in the limit turn the lottery contest into an all-pay auction with any bias-head-start combination of his choice. ${ }^{10}$ Consequently, first-best revenue is thereby also obtained in the lottery contest framework.

A meaningful restriction on score functions in the lottery contest is concavity. ${ }^{11}$ In the following subsection, we study revenue-maximizing concave score functions. Our approach is to show that any concave score function can be replaced by an appropriate affine bid transformation that induces weakly higher revenue, generalizing related results by Dasgupta and Nti (1998), [8], and Nti (2004), [33], to the n-player case with heterogeneous valuations. Hence, our revenue ranking

\footnotetext{
${ }^{9}$ Favoritism in an all-pay auction framework with incomplete information has been analyzed in Kirkegaard (2012), [23] and Kirkegaard (2013), [24].

${ }^{10}$ For example, consider score functions $s_{i}\left(x_{i}\right)=\left(\alpha_{i} x_{i}+\delta_{i}\right)^{r}$ for $r>0$ and the corresponding lottery CSF $\operatorname{Pr}_{i}^{L}=$ $\frac{s_{i}\left(x_{i}\right)}{\sum_{j} s_{j}\left(x_{j}\right)}$. Then, $\lim _{r \rightarrow \infty} \operatorname{Pr}_{i}^{L}=1$ if $\alpha_{i} x_{i}+\delta_{i}>\alpha_{j} x_{j}+\delta_{j}$ for all $j \neq i$ and $\lim _{r \rightarrow \infty} \operatorname{Pr}_{i}^{L}=0$ if $\alpha_{i} x_{i}+\delta_{i}<\alpha_{j} x_{j}+\delta_{j}$ for some $j$.

${ }^{11}$ Moreover, concavity of the score functions is sufficient for the existence of an equilibrium in pure strategies in the lottery framework. In contrast, for general convex score functions the set of (mixed-strategy) equilibria has not been fully characterized so far, see Alcalde and Dahm, [1], and Ewerhart (2015), [13], for some progress along these lines.
} 
for the lottery contest framework is robust in the sense that optimal biases revenue-dominate any combination of concave score functions.

\subsection{Concave score functions in the lottery contest framework}

We consider a lottery contest where arbitrary concave score functions, which include affine score functions considered in the rest of the paper as a special case, can be used to favor specific contestants. To be specific, we assume that, for each player $i$, there is a twice continuously differentiable score function $s_{i}$ with

$$
s_{i}(0) \geq 0, \quad s_{i}^{\prime}\left(x_{i}\right)>0, \quad \text { and } \quad s_{i}^{\prime \prime}\left(x_{i}\right) \leq 0 \quad \text { for all } x_{i}>0 .
$$

Player $i$ 's probability to win the lottery contest is given by

$$
\operatorname{Pr}_{i}^{C L}\left(x_{i}, x_{-i}\right)= \begin{cases}\frac{s_{i}\left(x_{i}\right)}{\sum_{j \in \mathcal{N}} s_{j}\left(x_{j}\right)}, & \text { if } \sum_{j \in \mathcal{N}} s_{j}\left(x_{j}\right)>0 \\ 0, & \text { otherwise. }\end{cases}
$$

As above for the affine case, we consider the equivalent game where each player $i$ chooses his score $y_{i} \in\left[s_{i}(0), \infty\right)$ and obtains payoff

$$
\tilde{\pi}_{i}\left(y_{1}, \ldots, y_{n}\right)=\frac{y_{i}}{Y} v_{i}-s_{i}^{-1}\left(y_{i}\right) \quad(\text { if } Y>0) .
$$

With $\tilde{\pi}_{i}$ being concave in $y_{i}, i$ 's best-response score is characterized by the first order condition

$$
\frac{Y-y_{i}}{Y^{2}} v_{i}-\left(s_{i}^{-1}\right)^{\prime}\left(y_{i}\right) \leq 0, \quad \text { with equality if } y_{i}>s_{i}(0) \text {, }
$$

which is a generalization of condition (1). As before, an effort profile $x_{1}, \ldots, x_{n}$ is a pure-strategy Nash equilibrium if and only if the corresponding score profile $y_{1}, \ldots, y_{n}$ satisfies (8) for each $i$.

The following result proves existence of a pure-strategy Nash equilibrium. Moreover, we find that any lottery contest with concave score functions can be replaced by a lottery contest with head starts and bias that induces the same equilibrium score profile, which corresponds to weakly higher efforts.

Proposition 6.1 The lottery contest with concave score functions $s_{1}, \ldots, s_{n}$ has a pure-strategy Nash equilibrium. If the efforts induced under $s_{1}, \ldots, s_{n}$ in such an equilibrium are $\tilde{x}_{1}, \ldots, \tilde{x}_{n}$, then the lottery contest with head start $\delta_{i}=s_{i}(0)$ and bias $\alpha_{i}=s_{i}^{\prime}\left(\tilde{x}_{i}\right)$ for $i \in \mathcal{N}$ induces equilibrium efforts $x_{1}, \ldots, x_{n}$ where $x_{i} \geq \tilde{x}_{i}$ for each $i$. 
Proof. We first prove existence of a pure-strategy Nash equilibrium. If $s_{i}(0)=0$ for all $i$, existence follows from Theorem 3 in Cornes and Hartley (2005), [7]. If $s_{i}(0)>0$ for at least one $i$, each player's payoff is continuous in all efforts and concave in his own effort. Moreover, as all efforts $x_{i}>v_{i}$ are strictly dominated for player $i$, we can without loss of generality restrict $i$ 's effort to the interval $\left[0, v_{i}\right]$, making the strategy set convex and compact. Hence, we can apply a standard existence theorem, for instance, Proposition 8.D.3 in Mas-Colell et al. (1995), [29].

Suppose $\tilde{x}_{1}, \ldots, \tilde{x}_{n}$ are equilibrium efforts under concave score functions. Accordingly, equilibrium scores $\tilde{y}_{i}=s_{i}\left(\tilde{x}_{i}\right)$ satisfy (8) for all $i$. Now, consider the lottery contest with head starts $\delta_{i}=s_{i}(0)$ and bias $\alpha_{i}=s_{i}^{\prime}\left(\tilde{x}_{i}\right)$. The scores $y_{1}, \ldots, y_{n}$ in the unique equilibrium of this contest are characterized by (1). Observe that $\frac{1}{\alpha_{i}}=\left(s_{i}^{-1}\right)^{\prime}\left(\tilde{y}_{i}\right)$. Hence, $\hat{y}_{i}$ satisfying (8) implies that $y_{i}=\hat{y}_{i}$ solves (1) for all $i$ and corresponds to the unique equilibrium under $(\delta, \alpha)$. Because $y_{i}=\hat{y}_{i}$, equilibrium efforts satisfy $s_{i}^{\prime}\left(\tilde{x}_{i}\right) x_{i}+s_{i}(0)=s_{i}\left(\tilde{x}_{i}\right)$. With $s_{i}$ being concave, we have $s_{i}\left(\tilde{x}_{i}\right) \geq s_{i}(0)+s_{i}^{\prime}\left(\tilde{x}_{i}\right) \tilde{x}_{i}$ and therefore $x_{i} \geq \hat{x}_{i}$ for all $i$.

Proposition 6.1 implies that allowing a contest organizer to apply any concave bid transformation cannot induce higher revenue than affine bid transformations involving bias and head starts. Hence, the result from Proposition 3.6 can be extended to any concave bid transformation.

Corollary 6.2 A lottery contest with optimal bias $\alpha^{*}$ maximizes revenue among all lottery contests with concave score functions.

\section{Conclusion}

Favoritism based on affine bid transformations involving bias and head starts can be a powerful instrument for generating additional revenue in contest games. However, the revenue potential of these instruments is highly dependent on the underlying contest framework. While in the lottery contest optimal biases induce substantial additional revenue because they level the playing field and therefore encourage the entry of formerly non-active weak players, head starts are less effective in this framework as favored agents substitute them for own effort. In the all-pay auction, by contrast, generically only two agents are active which severely limits the revenuepotential of using biases to induce additional entry of players. Head starts on the other hand are highly effective in the cut-throat competition of the all-pay auction because strong players have to exceed the highest head start of their rivals to maintain a positive winning probability. Hence, in the all-pay-auction framework it is revenue-maximizing to set the head start as large as possible without changing the effective ranking of the players. If head starts can be combined 
with biases, then the head start can be further increased up to the valuation of the strongest player by reducing the bias of the strongest player's active rival. In the end the optimal combination of bias and head starts in the all-pay auction resembles a take-it-or-leave-it offer to the strongest player. Thus, granting the contest organizer the right to design affine transformations of the bids, results in revenue extraction at the upper bound; however, at the same time competition is basically reduced to one player (the strongest) competing against a head start close to her valuation. Hence, in contrast to the lottery contest game, revenue is maximal in the all-pay auction under the optimal affine transformation, but the resulting framework does not look very competitive in the sense of high participation rates. In this sense, there is a trade-off between revenue-maximization on one side and participation in the contest game on the other side.

A pending issue is the complete equilibrium characterization of the all-pay auction with head starts because the algorithm of Siegel (2014), [37], is restricted to those equilibria, where only two players are active. Our equilibrium characterization of an all-pay auction with head starts involving three active players in the appendix constitutes a first step in this direction. A characterization of the complete equilibrium set will also be useful for other applications beyond contest design.

\section{A Appendix: Proof of Proposition 4.4}

We will first determine a Nash equilibrium under the given head starts, which is in mixed strategies. Let $y_{i}=x_{i}+\delta_{i}$ denoted the score of player $i$ if he invests effort $x_{i}$. Moreover, let $G_{i}$ denote a cumulative distribution function over scores $y_{i}$ for player $i$. As it is more convenient for our analysis, we will represent the equilibrium in terms of score distributions rather than effort distributions. The following lemma identifies equilibrium score distributions under an assumption on $\gamma$ that is less restrictive than the one stated in the proposition. ${ }^{12}$

Lemma A.1 Suppose $n=3, v_{1}>v_{2}>v_{3}$, and $v_{1}<v_{2}+v_{3}$. Then, for all $\gamma \in\left(0, v_{2}-v_{3}\right)$, the all-pay auction with head starts $\delta_{1}=0, \delta_{2}=v_{1}-v_{2}$, and $\delta_{3}=\delta_{2}+\gamma$ has a Nash equilibrium

\footnotetext{
${ }^{12}$ If $v_{1}<v_{2}+v_{3}$, then $\frac{v_{2}\left(v_{1}-v_{2}\right)\left(v_{2}-v_{3}\right)}{v_{1} v_{3}}<v_{2}-v_{3}$.
} 
with score distributions

$$
\begin{gathered}
G_{1}(y)=\left\{\begin{array}{ll}
0 & \text { if } y<\delta_{3}, \\
\frac{v_{1}}{v_{3} \hat{y}}\left(y-\delta_{3}\right) & \text { if } y \in\left[\delta_{3}, \hat{y}\right), \\
\frac{v_{1}}{v_{3} \hat{y}}\left(\hat{y}-\delta_{3}\right)=\frac{1}{v_{2}}\left(\hat{y}-\delta_{2}\right) & \text { if } y=\hat{y}, \\
\frac{1}{v_{2}}\left(y-\delta_{2}\right) & \text { if } y \in\left(\hat{y}, v_{1}\right], \\
1 & \text { if } y>v_{1},
\end{array} \quad G_{2}(y)= \begin{cases}0 & \text { if } y<\delta_{2}, \\
\frac{\hat{y}}{v_{1}} & \text { if } y \in\left[\delta_{2}, \hat{y}\right], \\
\frac{1}{v_{1}} y & \text { if } y \in\left(\hat{y}, v_{1}\right], \\
1 & \text { if } y>v_{1},\end{cases} \right. \\
\text { and } G_{3}(y)= \begin{cases}0 & \text { if } y<\delta_{3}, \\
\frac{1}{\hat{y}} y & \text { if } y \in\left[\delta_{3}, \hat{y}\right], \\
1 & \text { if } y>\hat{y},\end{cases}
\end{gathered}
$$

where

$$
\hat{y}:=\frac{\phi+\delta_{2}}{2}-\frac{1}{2} \sqrt{\left(\phi-\delta_{2}\right)^{2}-4 \phi \gamma}, \quad \hat{y} \in\left(\delta_{3}, v_{1}\right), \quad \text { and } \phi:=\frac{v_{1} v_{2}}{v_{3}} .
$$

Proof. We will show that no player $i$ has an incentive to deviate from mixing according to $G_{i}$, which yields zero expected payoff for each player.

Player 1: Given $G_{2}, G_{3}$, player 1's payoff from choosing score $y$ is

$$
G_{2}(y) G_{3}(y) v_{1}-y= \begin{cases}-y \leq 0 & \text { if } y<\delta_{3}, \\ \frac{\hat{y}}{v_{1}} \frac{1}{\hat{y}} y v_{1}-y=0 & \text { if } y \in\left[\delta_{3}, \hat{y}\right], \\ \frac{y}{v_{1}} v_{1}-y=0 & \text { if } y \in\left(\hat{y}, v_{1}\right], \\ v_{1}-y<0 & \text { if } y>v_{1} .\end{cases}
$$

Hence, randomizing according to $G_{1}$ is a best response.

Player 2: Let $u_{2}(y):=G_{1}(y) G_{3}(y) v_{2}-y+\delta_{2}$ denote player 2's payoff from score $y$. Scores $y \in \overline{\left(\delta_{2}, \delta_{3}\right)}$ and $y>v_{1}$ are clearly dominated as they yield $u_{2}(y)<0$ while $u_{2}\left(\delta_{2}\right)=0$. Note that $u_{2}(y)$ is continuous for $y \in\left[\delta_{3}, v_{1}\right]$. For $y \in\left[\hat{y}, v_{1}\right], u_{2}(y)=0$ and for $y \in\left[\delta_{3}, \hat{y}\right)$,

$$
u_{2}(y)=\frac{\phi}{\hat{y}^{2}}\left(y-\delta_{3}\right) y-y+\delta_{2} .
$$

As this is a strictly convex function, $u_{2}\left(\delta_{3}\right)=-\gamma<0$ and $u_{2}(\hat{y})=0$ implies $u_{2}(y)<0$ for all $y \in\left[\delta_{3}, \hat{y}\right)$. Consequently, $G_{2}$ is a best response to $G_{1}, G_{3}$.

Player 3: Let $u_{3}(y):=G_{1}(y) G_{2}(y) v_{3}-y+\delta_{3}$ denote player 3's payoff from score $y \geq \delta_{3}$ and note that this is a continuous function. For $y \in\left[\delta_{3}, \hat{y}\right], u_{3}(y)=\frac{v_{1}}{v_{3} \hat{y}}\left(y-\delta_{3}\right) \frac{\hat{y}}{v_{1}} v_{3}-y+\delta_{3}=0$ and for 
$y \in\left(\hat{y}, v_{1}\right]$

$$
u_{3}(y)=G_{1}(y) G_{2}(y) v_{3}-y+\delta_{3}=\frac{1}{\phi}\left(y-\delta_{2}\right) y-y+\delta_{3} .
$$

Because $u_{3}$ is strictly convex on $\left[\hat{y}, v_{1}\right], u_{3}(\hat{y})=0$ and $u_{3}\left(v_{1}\right)=v_{3}-v_{2}+\gamma<0$ implies $u_{3}(y)<0$ for all $y \in\left(\hat{y}, v_{1}\right]$. Hence, $G_{3}$ is a best response to $G_{1}, G_{2}$.

On the upper part of the equilibrium score support, only players 1 and 2 are active and mix as in the equilibrium of the two-player case. On the lower part, only players 1 and 3 are active. Player 1's equilibrium score distribution is continuous and atomless, whereas players 2 and 3 both have an atom at their respective head starts (i.e., they choose zero effort with strictly positive probability).

Lemma A.2 For any $\gamma \in\left(0, \frac{v_{2}\left(v_{1}-v_{2}\right)\left(v_{2}-v_{3}\right)}{v_{1} v_{3}}\right)$ the equilibrium of Lemma A.1 revenue-dominates any all-pay auction with head starts under which exactly two players are active.

Proof. As player 1's effort is uniformly distributed on $\left[\delta_{3}, \hat{y}\right]$ with probability $G_{1}(\hat{y})$ and on $\left[\hat{y}, v_{1}\right]$ with probability $1-G_{1}(\hat{y}), 1$ 's expected effort amounts to

$$
e_{1}:=G_{1}(\hat{y}) \frac{\delta_{3}+\hat{y}}{2}+\left(1-G_{1}(\hat{y})\right) \frac{\hat{y}+v_{1}}{2} .
$$

Similarly, player 2's effort is uniformly distributed on $\left[\hat{y}-\delta_{2}, v_{1}-\delta_{2}\right]$ with probability $1-G_{2}(\hat{y})=$ $\frac{v_{1}-\hat{y}}{v_{1}}$ and zero otherwise, resulting in expected effort

$$
e_{2}:=\frac{v_{1}-\hat{y}}{v_{1}} \cdot \frac{\hat{y}-\delta_{2}+v_{1}-\delta_{2}}{2}=\frac{\left(v_{1}-\hat{y}\right)\left(\hat{y}+v_{2}-\delta_{2}\right)}{2 v_{1}}
$$

To prove the proposition, we will show that $e_{1}+e_{2} \geq v_{1}-\frac{v_{2}}{2}+\frac{v_{2}^{2}}{2 v_{1}}$ if $\gamma \leq \frac{v_{2}\left(v_{1}-v_{2}\right)\left(v_{2}-v_{3}\right)}{v_{1} v_{3}}$. This implies that revenue is strictly greater than $v_{1}-\frac{v_{2}}{2}+\frac{v_{2}^{2}}{2 v_{1}}$ because in addition to players 1 and 2 also player 3 contributes to revenue.

In the two-player benchmark that yields revenue $v_{1}-\frac{v_{2}}{2}+\frac{v_{2}^{2}}{2 v_{1}}$, player 1's expected effort is $\frac{v_{1}+\delta_{2}}{2}$ and player 2's effort is $\frac{v_{2}^{2}}{2 v_{1}}$. Comparing these efforts to those in the three-player case, we find, after some rearranging,

$$
\begin{aligned}
e_{1}-\frac{v_{1}+\delta_{2}}{2} & =\frac{\left(\hat{y}-\delta_{2}\right) \gamma}{2 v_{2}}>0, \\
e_{2}-\frac{v_{2}^{2}}{2 v_{1}} & =-\frac{\left(\hat{y}-\delta_{2}\right)^{2}}{2 v_{1}}<0 .
\end{aligned}
$$


Hence, the presence of player 3 encourages player 1 to exert more effort while player 2 invests less. We now show that the net effect is positive, i.e.,

$$
e_{1}+e_{2}-\frac{v_{1}+\delta_{2}}{2}-\frac{v_{2}^{2}}{2 v_{1}}=\frac{\left(\hat{y}-\delta_{2}\right) \gamma}{2 v_{2}}-\frac{\left(\hat{y}-\delta_{2}\right)^{2}}{2 v_{1}} \geq 0
$$

This inequality can be reduced to $v_{1} \gamma-v_{2}\left(\hat{y}-\delta_{2}\right) \geq 0$, which, using the definition of $\hat{y}$, is equivalent to

$$
v_{2} \sqrt{\left(\phi-\delta_{2}\right)^{2}-4 \phi \gamma} \geq v_{2}\left(\phi-\delta_{2}\right)-2 v_{1} \gamma
$$

It can be shown that the RHS is positive for $\gamma \leq \frac{v_{2}\left(v_{1}\left(v_{2}-v_{3}\right)+v_{2} v_{3}\right)}{2 v_{1} v_{3}}$. After squaring both sides, we obtain

$$
-v_{2}^{2} \phi \gamma \geq v_{1}^{2} \gamma^{2}-v_{2}\left(\phi-\delta_{2}\right) v_{1} \gamma
$$

Substituting for $\delta_{2}$ and $\phi$, we find the above to be equivalent to $\gamma \leq \frac{v_{2}\left(v_{1}-v_{2}\right)\left(v_{2}-v_{3}\right)}{v_{1} v_{3}}$. Note that $\frac{v_{2}\left(v_{1}-v_{2}\right)\left(v_{2}-v_{3}\right)}{v_{1} v_{3}}<\frac{v_{2}\left(v_{1}\left(v_{2}-v_{3}\right)+v_{2} v_{3}\right)}{2 v_{1} v_{3}}$ because $v_{1}<v_{2}+v_{3}$; hence, $\gamma \leq \frac{v_{2}\left(v_{1}-v_{2}\right)\left(v_{2}-v_{3}\right)}{v_{1} v_{3} 1}$ is sufficient.

The assumption $v_{1}<v_{2}+v_{3}$ implies that the highest revenue over all equilibria with only two active players is given when only players 1 and 2 are active. What changes, if we introduce a third payer and give him head start $\delta_{3}$ with $\gamma$ as characterized in Lemma A.2? First of all, since $\delta_{3}>\delta_{2}$ the lower bound of the support of players 1's bidding distribution is pushed upwards (from $\delta_{2}$ to $\delta_{3}$ ). This increases the expected bid of player 1 . Player 2 now puts an atom on $\delta_{2}$ as before, but the lower bound of the support of his bidding distribution is also pushed upwards (from $\delta_{2}$ to $\hat{y}$ ). This, in fact, may lower his average expected bid as he counters the need to bid higher, if he enters, by a larger probability to stay inactive (i.e., increase the atom at $\delta_{2}$ ). Unequivocally, player 3's expected bid is positive. The further contributions of players 1 and 3 to total revenue dominate the possible reduction in Player 2's contribution, if $\gamma$ is chosen appropriately.

\section{References}

[1] J. Alchlde And M. Dahm: Rent seeking and rent dissipation: A neutrality result. Journal of Public Economics 94, 2010, pp. 1-7.

[2] L. Balafoutas AND M. SutTer: Affirmative action policies promote women and do not harm efficiency in the laboratory. Science 335, 2012, pp. 579-581.

[3] M.R. Baye, D. Kovenock, And C.G. DE VRIEs: Rigging the lobbying process: An application of the all-pay auction. American Economic Review 83(1), 1993, pp. 289-294. 
[4] M.R. BAYE, D. Kovenock, AND C.G. DE VRIES: The all-pay auction with complete information. Economic Theory 8(2), 1996, pp. 291-305.

[5] C. Calsamiglia, J. Franke, and P. Rey-Biel: The incentive effects of affirmative action in a real-effort tournament. Journal of Public Economics 98, 2013, pp. 15-31.

[6] L.C. Corchón: The theory of contests: A survey. Review of Economic Design 11, 2007, pp. 69-100.

[7] R. Cornes and R. Hartley: Asymmetric contests with general technologies. Economic Theory 26(4), 2005, pp. 923-946.

[8] A. Dasgupta and K.O. Nti: Designing an optimal contest. European Journal of Political Economy 14(4), 1998, pp. 587-603.

[9] M. Drugov and D. Ryvkin: Biased contests for symmetric players. Games and Economic Behavior, forthcoming, http://dx.doi.org/10.1016/j.geb.2016.02.003.

[10] J.F. English: Economy of Prestige: Prizes, Awards, and the Circulation of Cultural Value. Cambridge, MA: Harvard University Press, 2005.

[11] G.S. Epstein, Y. Mealem, and S. Nitzan: Political culture and discrimination in contests. Journal of Public Economics 1-2, 2011, pp. 88-93.

[12] G.S. Epstein, Y. Mealem, and S. Nitzan: Lotteries vs. all-pay auctions in fair and biased contests. Economics and Politics 25(1), 2013, pp. 48-60.

[13] C. EWERHART: Rent-seeking games and the all-pay auction. University of Zurich Working Paper No. 186, 2015.

[14] H. FANG: Lottery versus all-pay auction models of lobbying. Public Choice 112, 2002, pp. 351-371.

[15] R.H. Frank and P.J. Cook: The Winner-Take-All Society. New York: Virgin Books, 2010.

[16] J. Franke: Affirmative action in contest games. European Journal of Political Economy 28(1), 2012, pp. 105-118.

[17] J. Franke, C. Kanzow, W. Leininger, and A. Schwartz: Effort maximization in asymmetric contest games with heterogeneous contestants. Economic Theory 52(2), 2013, pp. 589-630. 
[18] J. Franke, C. Kanzow, W. Leininger, AND A. Schwartz: Lottery versus all-pay auction contests: A revenue dominance theorem. Games and Economic Behavior 83(2), 2014, pp. 116126.

[19] Q. Fu: A theory of affirmative action in college admissions. Economic Inquiry 44(3), 2006, pp. $420-428$.

[20] A.L. Hillman and J. Riley: Politically contestable rents and transfers. Economics and Politics 1(1), 1989, pp. 17-39.

[21] L. IMHOF AND M. KRÄKEL: Ex post unbalanced tournaments. RAND Journal of Economics 47(1), 2016, pp. 73-98.

[22] K. Kawamura and I. Moreno de Barreda: Biasing selection contests with ex-ante identical agents. Economics Letters 123(2), 2014, pp. 240-243.

[23] R. KirkegaARD: Favoritism in asymmetric contests: Head starts and handicaps. Games and Economic Behavior 76(1), 2012, pp. 226-248.

[24] R. KirkegaARD: Handicaps in incomplete information all-pay auctions with a diverse set of bidders. European Economic Review 64(0), 2013, pp. 98-110.

[25] K.A. KonRAD: Investment in the absence of property rights; the role of incumbency advantages. European Economic Review 46(8), 2002, pp. 1521-1537.

[26] K.A. Konrad: Strategy and Dynamics in Contests. Oxford University Press, 2009.

[27] E. Krasnokutskaya and K. Seim: Bid Preference Programs and Participation in Highway Procurement Auctions. American Economic Review 101, 2011, pp. 2653-2686.

[28] S. Li and J. Yu: Contests with endogeneous discrimination. Economics Letters 117(3), 2012, pp. 834-836.

[29] A. Mas-Colell, M.D. Whinston, and J.R. Green: Microeconomic Theory. Oxford University Press, 1995.

[30] A. Matros and A. Possajennikov: Tullock contests may be revenue superior to auctions in a symmetric setting. Economics Letters 142, 2016, pp. 74-77.

[31] Y. Mealem and S. Nitzan: Discrimination in contests: a survey. Review of Economic Design, forthcoming. 
[32] M. Niederle, L. Vesterlund and C. Segal: How costly is diversity? Affirmative action in light of gender differences in competitiveness. Management Science 59(1), 2013, pp. 1-16.

[33] K.O. NTI: Maximum efforts in contests with asymmetric valuations. European Journal of Political Economy 20(4), 2004, pp. 1059-1066.

[34] D. Pérez-Castrillo and D. Wettstein: Discrimination in a model of contests with incomplete information about ability. International Economic Review 57(3), 2016, pp. 881-914.

[35] C. Seel and C. Wasser: On optimal head starts in all-pay auctions. Economics Letters 124(2), 2014, pp. 211-214.

[36] E. Segev and A. Sela: Sequential all-pay auctions with head starts. Social Choice and Welfare 43(4), 2014, pp. 893-923.

[37] R. SIEGEL: Asymmetric contests with head starts and non-monotonic costs. American Economic Journal: Microeconomics 6(3), 2014, pp. 59-105.

[38] G. Tullock: Efficient rent seeking. In J. Buchanan, R. Tollison, and G. Tullock (eds.): Towards a Theory of the Rent-Seeking Society. A \& M University Press, 1980, pp. 97-112.

[39] M. Vojnović: Contest Theory. Incentive Mechanisms and Ranking Methods. Cambridge University Press, 2016.

[40] C. WASSER: Incomplete information in rent-seeking contests. Economic Theory 53(1), pp. 239-268. 